\title{
Postpartum Posterior Reversible Encephalopathy Syndrome - A Case that can Press Hard an Obstetrician
}

\author{
FAHMIDARASHID ${ }^{1}$, MD. ABDUS SATTAR ${ }^{1}$
}

\begin{abstract}
:
Posterior reversible encephalopathy syndrome (PRES) is a recently described clinicoradiologic entity that is associated with several medical conditions like hypertensive encephalopathy and eclampsia. It present with headache, confusion, visual disturbances or blindness, and seizures. Parieto-occipital white matter changes due to vasogenic edema can be observed on imaging modalities. It rarely occurs without seizures. There have been reports about PRES associated with pregnancy, especially peripartum. It is often, but not always, associated with high blood pressure. The pathophysiology of PRES is not still clear. Here we report a 23-yearold primigravida with unremarkable antenatal period but complicated by PRES with seizures at her $5^{\text {th }}$ postpartum day. Postictal findings reported headache and magnetic resonance imaging (MRI) findings suggested that PRES were evident. Clinical improvement with complete resolution without any complications was observed on the 8th post operative day with supportive treatment. This case report highlights the importance of awareness, prompt diagnosis and treatment to improve the outcome in this potentially life-threatening, but reversible condition.
\end{abstract}

Key words: Cesarean delivery, posterior reversible encephalopathy syndrome, postpartum seizures, Eclampsia.

\section{Introduction:}

Convulsions in immediate post partum period are a diagnostic dilemma. There are so many conditions that may be responsible and also presentations are so varied that it is difficult to diagnose what might be the cause of convulsion. Convulsions in early puerperium are treated as eclampsia unless proved otherwise. But other causes of convulsion should also be kept in mind and investigated thereby. Posterior reversible encephalopathy syndrome (PRES) is a cliniconeuroradiologic entity described by Hinchey et al. in $1996^{1}$ PRES is a clinical and radiological syndrome which is also known as reversible posterior cerebral edema syndrome, hyperperfusion encephalopathy, or brain capillary leak syndrome ${ }^{2}$. Many causes of PRES have been reported, which includes hypertensive encephalopathy, preeclampsia, eclampsia, acute or chronic renal failure, hemolytic uremic syndrome, immunosuppressants such as cytotoxic drug (e.g. cyclosporine and tacrolimus), acute intermittent porphyria, thrombotic thrombocytopenic purpura and systemic lupus erythematosus (SLE), blood transfusion and electrolyte disturbances ${ }^{3-5}$. Preeclampsia and eclampsia are the most common causes of PRES. Prompt recognition and treatment are crucial to avoid the permanent damage leading to sequelae and even mortality.

There are reports of various cases of PRES developed during peripartum ${ }^{1,3,5-10}$.

Even though PRES is increasingly recognized and reported in the current literature, its incidence is still obscure. In this case report, we present woman complicated by PRES after delivery with seizures due to sudden rise of blood pressure at her $5^{\text {th }}$ post operative day.

1. Assistant Professor, Department of Gynae and Obs ,Chittagong Medical college, Bangladesh

2. Associate Prof. Dept. Of Medicine, Chittagong Medical College. 


\section{Case Report:}

A 23-year old primigravida at the 39th week of gestation was admitted to hospital for elective cesarean section due to cephaliopelvic disproportion. Her entire pregnancy period was uneventful. Throughout pregnancy her blood pressure varied from 100/70 to $110 / 75 \mathrm{~mm}$ of $\mathrm{Hg}$. Her routine blood and urine investigations were done periodically during ante natal period, which were within normal range. A subarachnoid block with $2 \mathrm{ml}$ of $0.5 \%$ bupivacaine was given at L3-L4 space under aseptic precautions and LUCS was done and a female healthy baby was delivered weighing $3.1 \mathrm{~kg}$ with good APGAR (8-9 at 1 $\mathrm{min}$ ) score. There was no rise in BP after delivery and no accompanying relevant symptom or sign thereafter. It took 45 mins to complete the surgery and total blood loss was around $800 \mathrm{ml}$. Remaining post operative course was uneventful and she was shifted to recovery ward for observation.

On her $2^{\text {nd }}$ post operative day, as she was looking completely alright, she was discharged with usual medication and advice. On $5^{\text {th }}$ POD morning she went to a near facility with the complaints of severe headache and sleeplessness for last 4 days due to pain and taking care of the baby. She had a convulsion at home with frothy secretion in her mouth. Doctor advised her to shift to hospital immediately and on her way to hospital she had $2^{\text {nd }}$ convulsion. At hospital in front of attending doctor she had $3^{\text {rd }}$ convulsion at around which was tonic and clonic in nature and persisted for about 30 secs. The BP recorded at that moment was high (150/95 mm of $\mathrm{Hg}$ ). Her routine investigation revealed -Hemoglobin - $10.8 \mathrm{~g} / \mathrm{dl}$, total leucocyte count - $15 \times 103 / 1 / 4 \mathrm{~L}$, Platelets - $170 \times 103 /$ $1 / 4$ L, RFT- Normal, LFT- Normal, Clotting parametersNormal and Urine R E - Normal.

Assuming as a case of eclampsia, she was promptly treated with magnesium sulfate loading dose over 15 min and diazepam intravenous (IV). In the postictal phase, her pulse rate was 114 beats/min, BP 150/90 $\mathrm{mm}$ of $\mathrm{Hg}$ and oxygen saturation was $100 \%$ with oxygen supplement. Bed side heat coagulation test was done and found negative.

Neurological examinations were carried out after the convulsion episode by neurologist and found essentially normal without any sensory or motor deficit. MRI of Brain was done and she was shifted to HDU for further management. MRI findings showed bilateral hyper intensity in fronto-parietal, occipital cortical and subcortical white matter with increased apparent diffusion coefficient values in these regions suggestive of vasogenic edema. In HDU, her treatment consisted of continuous BP monitoring, Sodium phenytoin, 15 $\mathrm{mg} / \mathrm{kg}$ orally and injection oradexone iv. She was closely monitored with all the necessary HDU protocols including periodic neurological assessment and biochemical profile. She stayed 72 hours at HDU and had no further convulsions and was getting better in course of time and discharged on $8^{\text {th }}$ POD without any neurological sequelae. During discharge she was advised to take phenytoin $100 \mathrm{mg}$ orally twice daily for 6 months.

A follow up MRI after 4 weeks showed complete resolution of the high signal foci previously noted. Now 7 months after her confinement, she is completely alright and happy with her healthy kid. She stopped phenytoin 1 month ago. She had no neurological problem till today.

\section{Discussion:}

Posterior reversible encephalopathy syndrome (PRES) is a compilation of various neurologic signs and symptoms which includes headache, nausea, vomiting, altered mental status, seizure, visual disturbance, focal neurologic deficits, stupor and coma $^{3,11}$. Clinical and radiological features of PRES include headache, encephalopathy, seizures, cortical visual changes, and parieto-occipital white matter edema visualized on neuroimaging modalities ${ }^{12}$. In this case, headache and convulsion in early post partum period without proteinuria might be due to cerebral venous sinus thrombosis, subarachnoid hemorrhage and postdural punctures seizures in the postpartum period $^{13}$. The diagnosis of PRES was made following the typical radiological findings of bilateral, symmetrical cortical and subcortical vasogenic edema in combination with the characteristic presenting features.

The pathophysiological mechanism underlying PRES is still vague. It may be related to disordered cerebral autoregulation and endothelial dysfunction. The combination of acute hypertension and endothelial damage can lead to vasogenic edema elicited by the forced leakage of serum through capillary walls and into the brain interstitium ${ }^{14}$. The reason for the primary involvement of posterior brain regions is not well understood. One possibility may be the regional heterogeneity of the sympathetic innervation of the intracranial arterioles. This is explained by better 
autoregulation of the anterior circulation due to better sympathetic innervations as compared to the posterior circulation ${ }^{15}$. Nevertheless, acute hypertension can lead to hyperperfusion and edema in the posterior circulation in PRES.

The key investigation for the diagnosis of PRES is cerebral MRI and not cerebral CT since the latter is often normal or nonspecific ${ }^{15}$. The typical MRI findings in T2-weighted and FLAIR sequences showed hyper intense foci located bilaterally at the grey-white junctions, involving sub cortical white matter of most parts of brain; although it was earlier believed to affect the posterior parietal and occipital lobes mainly ${ }^{15,16}$.

Owing to the heterogeneity of clinical settings and relative lack of data on PRES, treatment recommendations are somewhat limited. The initial goal in malignant hypertension is to rapidly lower the diastolic pressure to about 100 to $105 \mathrm{~mm}$ of $\mathrm{Hg}$. More aggressive blood pressure control may reduce the blood pressure below the autoregulatory range and ischemic events such as stroke or coronary disease may appear ${ }^{17}$. In most cases of PRES, neurological symptoms and cerebral lesions disappear completely within days to weeks after control of blood pressure. Recommendations for the treatment of eclampsia differ from PRES in other clinical settings. Delivery of the baby and placenta is often sufficient. Magnesium therapy should be initiated as soon as eclampsia or PRES in pregnancy is suspected, since it treats both seizures and hypertension ${ }^{18}$.

The prognosis of PRES is usually benign. Regardless of etiology, hypertension is a feature in the vast majority of PRES patients. Most investigators believe that hypertensive encephalopathy and preeclampsia share similar mechanisms. Clinical improvement always follows the treatment of elevated blood pressure.

Posterior reversible leukoencephalopathy syndrome may be a diagnostic and therapeutic challenge when it develops in eclampsia and preeclampsia. The association of PRES with toxemia of pregnancy is well-established ${ }^{19}$. PRES can occur, within several weeks after delivery and the clinical presentation is often confusing 20 .

Global incidence of PRES is unknown. It has been reported in patients aged 4-90 years, although the most cases occur in age group of 39-47 years with a marked female predominance. ${ }^{21}$ Mechanical ventilation is required in $35-40 \%$ of patients with PRES for 3-7 days ${ }^{22}$. Recurrence are reported to be occurring in $6 \%$ cases. Permanent neurologic damage occurs due to ischemia (10-23\%) and hemorrhage (5-17\%). Death is reported in $15 \%$ cases $^{23}$.

In our case, no focal deficits or visual abnormalities were detected before the delivery. In most cases of PRES, neurological symptoms and cerebral lesions disappear with treatment. Control of blood pressure is vital to avoid irreversible damage to central nervous system.

A clinical report of parturient with PRES after the delivery of baby is presented. It emphasizes the need for early diagnosis and prompt treatment to avoid any short and long term neurological sequelae including death in a reversible condition like PRES.

\section{Conflict of Interests}

There is no conflict of interests for any author regarding this paper.

\section{References:}

1. Hinchey J, Chaves C, Appignani B, Breen J, Pao L, Wang A, A reversible posterior leukoencephalopathy syndrome. N Engl J Med. 1996;334:494-500.

2. L. H. Calabrese, D. W. Dodick, T. J. Schwedt, and A. B. Singhal, "Narrative review: reversible cerebral vasoconstriction syndromes," Annals of Internal Medicine. 2007;146(1):34-44.

3. Long TR, Hein BD, Brown MJ, Rydberg CHWass CT. Posterior reversible encephalopathy syndrome during pregnancy: seizures in a previously healthy parturient. J Clin Anesth. 2007;19(2):145-8.

4. Cosottini M, Lazzarotti G, Ceravolo R, Michelassi MC, Canapicchi R, Murri L. Cyclosporin related posterior reversible encephalopathy syndrome (PRES) in non transplant patient: a case report and literature review. Eur J Neurol. 2003;10(4):461-2.

5. Gimovsky ML, Guzman GM, Koscica KL, Nazir MA, Ross DE. Posterior reversible encephalopathy with late postpartum eclampsia and short-term memory loss. J Reprod Med. 2010;55(1-2):71-4.

6. Fujiwara Y, Higaki H, Yamada T, Nakata Y, Kato $\mathrm{S}$, Yamamoto $\mathrm{H}$, et al. Two cases of reversible 
posterior leukoencephalopathy syndrome, one with and the other without pre-eclampsia. $\mathrm{J}$ Obstet Gynaecol Res 2005; 31(6):520-6.

7. Onrubia X, Lluch-Oltra A, Armero R, Higueras R, Sifre C, Barbera M. Posterior reversible encephalopathy syndrome after cesarean delivery. Anesth Analg 2007; 104(3):746-7.

8. Servillo G, Striano P, Striano S, Tortora F, Boccella P, De Robertis E, et al. Posterior reversible encephalopathy syndrome (PRES) in critically ill obstetric patients. Intensive Care Med. 2003;29(12):2323-6.

9. Tagg WG, Razdan RS, Swain FR, Gagliardi JA, Chaddha SK. Posterior reversible encephalopathy syndrome following a cesarean delivery: case report and literature review. Conn Med. 2008;72(5):267-9.

10. Ko ML, Pan HS, Huang LW, Hwang JL, Chen SC, Chang JZ. Posterior reversible encephalopathy syndrome in a pregnant woman. Taiwan J Obstet Gynecol 2008;47(1):98-100.

11. Pula JH, Eggenberger E. Posterior reversible encephalopathy syndrome. Curr Opin Ophthalmol. 2008;19(6):479-84.

12. W. S. Bartynski and J. F. Boardman, "Distinct imaging patterns and lesion distribution in posterior reversible encephalopathy syndrome," American Journal of Neuroradiology. 2007; 28(7): 1320-1327.

13. Shearer VE, Jhaveri HS, Cunningham FG. Puerperal seizures after postdural puncture headache. Obstet Gynecol. 1995;85:255-60.

14. R. B. Schwartz, S. K. Feske, J. F. Polak et al., "Preeclampsia-eclampsia: clinical and neuroradiographic correlates and insights into the pathogenesis of hypertensive encephalopathy," Radiology.2000;(2):371-376.

15. W. S. Bartynski, "Posterior reversible encephalopathy syndrome — part 1 : fundamental imaging and clinical features," American Journal of Neuroradiology. 2008; 29(6):1036-1042.

16. Casey SO, Sampaio RC, Michel E, Truwit CL. Posterior reversible encephalopathy syndrome: Utility of fluid-attenuated inversion recovery MR imaging in the detection of cortical and subcortical lesions. AJNR Am J Neuroradiol. 2000;21:1199-206.

17. C. Roth and A. Ferbert, "Posterior reversible encephalopathy syndrome: long-term follow-up," Journal of Neurology, Neurosurgery and Psychiatry. 2010; 81(7):773-777.

18. P. Striano, S. Striano, F. Tortora et al., "Clinical spectrum and critical care management of posterior reversible encephalopathy syndrome (PRES)," Medical Science Monitor. 2005; 11(11):CR549-CR553.

19. Thackeray EM, Tielborg MC. Posterior reversible encephalopathy syndrome in a patient with severe preeclampsia. Anesth Analg. 2007;105:184-6.

20. Bartynski WS, Sanghvi A. Neuroimaging of delayed eclampsia. Report of 3 cases and review of the literature. J Comput Assist Tomogr. 2003;27:699-713.

21. Legriel S, Pico F, Azoulay E. Understanding posterior reversible encephalopathy syndrome. In: Vincet JL, editor. Annual Update in Intensive Care and Emergency Medicine. Springer Berlin Heidelberg. 2011;1:631-53.

22. Burnett MM, Hess CP, Roberts JP, Bass NM, Douglas VC, Josephson SA. Presentation of reversible posterior leukoencephalopathy syndrome in patients on calcineurin inhibitors. Clin Neurol Neurosurg. 2010;112:886-91.

23. Lee VH, Wijdicks EF, Manno EM, Rabinstein AA. Clinical spectrum of reversible posterior leukoencephalopathy syndrome. Arch Neurol. 2008;65:205-10. 